Jansen, D.L., Rijken, M., Kaptein, A.A., Boeschoten, E.W., Dekker, F.W., Groenewegen, P.P. The role of social support in dialysis patients' feelings of autonomy and self-esteem: is support more beneficial for patients with specific illness perceptions? Families, Systems \& Health: 2014, 32(3), 313-327

\begin{tabular}{|l|l|}
$\begin{array}{l}\text { Postprint } \\
\text { Version }\end{array}$ & 1.0 \\
\hline Journal website & $\underline{\text { http://psycnet.apa.org/psycinfo/2014-24855-001/ }}$ \\
\hline Pubmed link & $\underline{\text { http://www.ncbi.nlm.nih.gov/pubmed/24933040 }}$ \\
\hline DOI & 10.1037/fsh0000028
\end{tabular}

This is a NIVEL certified Post Print, more info at http://www.nivel.eu

\title{
The Role of Social Support in Dialysis Patients’ Feelings of Autonomy and Self-Esteem: Is Support More Beneficial for Patients With Specific Illness Perceptions?
}

\author{
Jansen, Daphne L. PhD ${ }^{1}$; RiJken, Mieke PhD ${ }^{1}$; Kaptein, Ad A. PhD²; Boeschoten, \\ Elisabeth W. MD, PhD ${ }^{3}$; DeKKer, Friedo W. PhD ${ }^{4}$; Groenewegen, Peter P. PhD ${ }^{5}$ \\ ${ }^{1}$ NIVEL, Netherlands Institute for Health Services Research, Utrecht, The Netherlands \\ ${ }^{2}$ Department of Medical Psychology, Leiden University Medical Centre (LUMC) \\ ${ }^{3} \mathrm{HMI}$, Hans Mak Institute, Naarden, The Netherlands \\ ${ }^{4}$ Department of Clinical Epidemiology, Leiden University Medical Centre (LUMC) \\ ${ }^{5}$ NIVEL, Netherlands Institute for Health Services Research, and Department of Sociology \\ and the Department of Human Geography, Utrecht University, Utrecht, The Netherlands
}

\begin{abstract}
The purpose of this study was to investigate whether effects of various types of support on dialysis patients' perceived autonomy and self-esteem depend on patients' perceived concerns and personal control regarding their illness. One hundred sixty-six patients completed written questionnaires. Main and interaction effects of support, concern, and personal control on autonomy and self-esteem were examined using linear regression analyses. General emotional support was positively related to autonomy in highly concerned patients ( $p<$ $.05)$. Overprotection was negatively associated with autonomy $(p<.05)$, and this association was stronger in patients with high perceived personal control ( $p$ $<.01)$. A positive main effect of general emotional support $(p<.05)$ and a negative main effect of overprotection $(p<.01)$ on self-esteem were observed. The role of support in dialysis patients' autonomy appears to depend on patients' illness perceptions, whereas the role of support in patients' self-esteem does not. These findings suggest that dialysis patients' personal views about their illness can provide insight into whether patients could benefit from support, and that the provision of support should be tailored to patients' individual needs.
\end{abstract}

People with end-stage renal disease (ESRD, Chronic Kidney Disease stage 5) require renal replacement therapy - dialysis or transplantation - to sustain life. In 2008, the overall prevalence of renal replacement therapy for ESRD across Europe-among all 
Jansen, D.L., Rijken, M., Kaptein, A.A., Boeschoten, E.W., Dekker, F.W., Groenewegen, P.P. The role of social support in dialysis patients' feelings of autonomy and self-esteem: is support more beneficial for patients with specific illness perceptions? Families, Systems \& Health: 2014, 32(3), 313-327

registries reporting to the ERA-EDTA Registry—was 644 per million population (Stel et al., 2011). Dialysis in particular is burdensome and intrusive. Compared with general population samples, patients with ESRD on dialysis experience, besides impaired physical functioning, impaired mental and social functioning (e.g., Khan et al., 1995; Merkus et al., 1997; Molsted, Prescott, Heaf, \& Eidemak, 2007). Results of a Swedish study demonstrated that more than $50 \%$ of dialysis patients reported stressors with respect to work and leisure time (Ekelund \& Andersson, 2007). Restrictions on the quantity and quality of daily activities might impede people's feelings of autonomy. A Dutch study showed that patients on dialysis, on average, have moderate feelings of autonomy, which indicates that they do not often feel that they can do the things they wish to do in everyday life, because of their health condition or otherwise (Jansen, Rijken, Heijmans, \& Boeschoten, 2010). According to Deci and Ryan's Self-Determination Theory (SDT; Deci \& Ryan, 1985) autonomy is one of the basic psychological needs for optimal functioning. When the fulfillment of the need for autonomy is hindered, one's experience of self-worth is also damaged, leading to either insecure or low self-esteem (Ryan \& Brown, 2003).

Recognizing the importance of dialysis patients' quality of life, it is interesting to know why patients, who have a comparable clinical status, differ in the extent to which they feel autonomous and experience self-esteem. By gaining insight into this matter, starting points can be generated for promoting patients' feelings of autonomy and self-esteem. Previous research suggests that renal patients' social environment plays an important role, by supporting patients in their efforts to carry on with daily activities (Heijmans \& Rijken, 2004), which in turn might support patients' sense of autonomy. Support may, however, also undermine patients' feelings of autonomy as well as their self-esteem, in case the support is perceived as controlling or overprotective.

Significant relationships have been found between different types of support and well-being (Baard, Deci, \& Ryan, 2004; Buunk, Berkhuysen, Sanderman, Nieuwland, \& Ranchor, 1996; Cohen \& Wills, 1985; Deci, La Guardia, Moller, Scheiner, \& Ryan, 2006; Joekes, Van Elderen, \& Schreurs, 2007; Kasser \& Ryan, 1999; Sarason, Sarason, \& Gurung, 1997; Thompson \& Sobolew-Shubin, 1993). Studies in patients with ESRD also have demonstrated associations between social support and depressive affect, quality of life (Patel, Peterson, \& Kimmel, 2005), and even mortality (Thong, Kaptein, Krediet, Boeschoten, \& Dekker, 2007). However, some studies did not demonstrate relationships between support and wellbeing in patient populations (e.g., Buunk et al., 1996; De Ridder, Schreurs, \& Kuijer, 2005). The question arises why social support sometimes does and sometimes does not work. According to the stress-buffering model, social support is only beneficial for those suffering adversity but does not play a role for those without highly stressful demands (Cohen, 2004). Moreover, not all types of support may be equally beneficial in face of the demands. Cohen (2004) found that, when types of perceived support were broken down, emotional support worked in the face of a variety of stressful events, whereas other types of support (e.g., instrumental, informational) responded to specific needs elicited by an event.

Opposed to the stress-buffering model, the main effect model of social support argues that support is beneficial irrespective of whether one is under stress (see Cohen \& Wills, 1985). 
Jansen, D.L., Rijken, M., Kaptein, A.A., Boeschoten, E.W., Dekker, F.W., Groenewegen, P.P. The role of social support in dialysis patients' feelings of autonomy and self-esteem: is support more beneficial for patients with specific illness perceptions? Families, Systems \& Health: 2014, 32(3), 313-327

Having a chronic illness can be viewed as a potentially stressful life event. Tijhuis, Flap, Foets, and Groenewegen (1995) investigated the stress-buffering effect of social support on duration and disabilities of illness in a sample of chronic ill people, taking the illness itself as a stressor. Their results did not reveal systematic buffer effects. The authors concluded that the diseases studied might not be severe or threatening enough for the buffer effect to function. The way people view their illness might be a valuable indication of the extent to which people experience their illness as a stressful event, and consequently of whether or not support could be helpful. Patients' beliefs about their illness are the central concepts of Leventhal's Common Sense Model (CSM; Leventhal, Meyer, \& Nerenz, 1980; Leventhal, Nerenz, \& Steele, 1984), which is a self-regulation model of health threat. According to this model, people make sense of a health threat by developing their own cognitive (e.g., beliefs about control or cure: extent to which the illness can be controlled or cured through treatment and behaviors, and beliefs about consequences: expected effects of an illness on physical, social and psychological well-being) and emotional representations of that threat (e.g., fear and worry; Cameron \& Moss-Morris, 2004). In particular for those who believe that they cannot personally control their illness and those who are highly concerned about their illness, life with ESRD is likely to be stressful. The question we address in the present study is as follows: What is the role of social support, including overprotection, in ESRD patients' feelings of autonomy and self-esteem, and does the association depend on patients' levels of concern and perceived personal control regarding their illness? Or in other words, is social support more beneficial for some than for others?

\section{Emotional Support and Autonomy}

Based on the stress-buffering model, we expect a positive relationship between emotional support (including general emotional support, e.g., are affectionate toward you, and problem-oriented emotional support, e.g., give you a nudge in the right direction) on the one hand and autonomy on the other hand, but only in patients who report high concern and low personal control. We thus predict that patients with low levels of concern and high levels of personal control are not dependent on emotional support to act according to their wishes and values, and thus will not benefit from this kind of support. Consequently, we do not expect any main effects of emotional support on autonomy. This latter hypothesis follows from the idea that the experience of emotional support is not a universal necessity for experiencing a sense of autonomy, and that experiencing a sense of autonomy may be more related to autonomy-supporting interactions rather than to the experience of emotional support.

\section{Emotional Support and Self-Esteem}

In line with the previous hypotheses, we hypothesize a positive relationship between problem-oriented emotional support and self-esteem in patients who report high concern and low personal control. So here too, we expect no main effects on selfesteem. We however expect that general emotional support is positively related to self-esteem in all patients. This assumption follows from the general emphasis of self-esteem theories on the importance of perceptions of belongingness and relatedness to people's self-esteem (Deci \& Ryan, 2000; Leary \& Baumeister, 2000). Experienced general emotional support might reflect satisfaction of the general need for belongingness or relatedness, and therefore contribute to people's self-esteem. In 
Jansen, D.L., Rijken, M., Kaptein, A.A., Boeschoten, E.W., Dekker, F.W., Groenewegen, P.P. The role of social support in dialysis patients' feelings of autonomy and self-esteem: is support more beneficial for patients with specific illness perceptions? Families, Systems \& Health: 2014, 32(3), 313-327

addition, we expect that this relationship will be stronger in patients who report high levels of concern and low levels of personal control.

\section{Overprotection and Autonomy, Self-Esteem}

Finally, we expect a negative association between experienced overprotection on the one hand and autonomy and self-esteem on the other hand in all patients. Negative main effects are expected because overprotection refers to unwanted help, which is likely to detract from one's feelings of autonomy and self-esteem. In addition, we hypothesize that this relationship will be stronger in people who report low levels of concern and high levels of personal control. This latter hypothesis results from the assumption that these patients are less in need of help to maintain their sense of autonomy and self-esteem and unwanted help will therefore have an aggravating impact. Summarized, this brings us to the following hypotheses:

1. General and problem-oriented emotional support are positively related to autonomy in patients who report high levels of concern and low levels of personal control (buffering effects).

2a. Problem-oriented emotional support is positively related to self-esteem in patients who report high levels of concern and low levels of personal control (buffering effects).

2b. General emotional support is positively related to self-esteem in all patients (main effect), and this relationship is stronger in patients who report high levels of concern and low levels of personal control (buffering effects).

3. Overprotection is negatively related to autonomy and self-esteem in all patients (main effects), and these relationships are stronger in patients who report low levels of concern and high levels of personal control (buffering effects).

These hypotheses were tested in a study among patients with ESRD on dialysis, because this patient group and their significant others are required to actively deal with the illness demands on a daily basis.

\section{METHOD}

\section{Study Sample}

Patients on dialysis who participated in the longitudinal Netherlands Cooperative Study on the Adequacy of Dialysis phase 2 (NECOSAD-2; Termorshuizen et al., 2003; Timmers et al., 2008) were invited to participate in the present study. The NECOSAD-2 study was approved by the Medical Ethical Committees of the participating dialysis centers and hospitals. Inclusion criteria were as follows: being older than 18 years, and having no previous history of renal replacement therapy. Since 1997, more than 2,000 dialysis patients were recruited on an ongoing basis in more than 40 dialysis centers and hospitals in The Netherlands. 1

Within NECOSAD-2 clinical (medical records) and quality of life (self-report) data are collected every six months. For the present study, an additional survey was conducted in March/April 2006 among the 248 being followed in the NECOSAD-2 cohort in January 2006. Twenty-seven dialysis centers and hospitals, participating in the NECOSAD-2 study, contributed to the implementation of the present study. The questionnaires were handed out by the nurses in the hospitals. Patients filled in the 
Jansen, D.L., Rijken, M., Kaptein, A.A., Boeschoten, E.W., Dekker, F.W., Groenewegen, P.P. The role of social support in dialysis patients' feelings of autonomy and self-esteem: is support more beneficial for patients with specific illness perceptions? Families, Systems \& Health: 2014, 32(3), 313-327

questionnaires at home or in the hospital and returned them by mail. Out of 248 patients contacted, 166 patients returned the questionnaire (response rate 67\%).

\section{Measures}

\section{Dependent variables.}

Perceived autonomy was assessed with two items derived from the autonomy scale of the CASP-19 (Hyde, Wiggins, Higgs, \& Blane, 2003). The two items were combined on the basis of their high factor loadings on one factor (both factor loadings: 0.81, variance explained: 66\%) to assess 'global autonomy' (I can do the things that I want to do, I feel that I can please myself what I can do). Items were scored on a 4-point scale ( $0=$ never, 1 = sometimes, 2 = not so often, $3=$ often $)$. Global autonomy scores are expressed as average scores based on the two items. Higher scores signify a higher level of perceived autonomy.

State self-esteem was measured with the Current Thoughts Scale (Heatherton \& Polivy, 1991), which comprises 20 items (e.g., I am worried about what other people think of me [reverse-scored item]). Items were rated on a 5-point scale (1 = not at all, 2 = a little bit, $3=$ somewhat, $4=$ very much, $5=$ extremely). Scores are summed across individual ratings with higher scores representing a higher level of state selfesteem. The Cronbach's alpha (i.e., internal consistency) for the scale in the current study was 0.88 .

\section{Independent variables.}

Social support was measured with the scales 'daily oriented emotional support' and 'problem-oriented emotional support' of the Social Support List-Interactions (Van Sonderen, 1993). This is a self-report questionnaire that has been designed and validated in The Netherlands. The Social Support List-Interactions measures the experienced amount of support received from people in the close environment, such as members of the family, friends, and so forth.

The scales 'daily oriented emotional support' and 'problem-oriented emotional support' consist of four items (e.g., Does it ever happen to you that people cuddle/hug you?) and eight items (e.g., Does it ever happen to you that people perk you up or cheer you up?) respectively. The items are all answered on a 4-point scale $(1=$ seldom or never, $2=$ now and then, $3=$ regularly, $4=$ very often $)$. Scores are summed across items with higher scores indicating greater levels of experienced support.

The internal consistency of the 'daily oriented emotional support' scale and 'problem-oriented emotional support' scale in this study were 0.82 and 0.92 , respectively. Hereafter, we will refer to 'general emotional support' instead of 'daily oriented emotional support.'

Overprotection by significant others was measured using the Overprotection Scale for Adults (OPSA; Thompson \& Sobolew-Shubin, 1993), which is a validated selfreport questionnaire. This scale measures the patient's perceptions concerning overprotection by members of the family and friends. The scale consists of 18 items (e.g., The people around me do not let me do the things I could do myself). All items are answered on a 4-point scale (1 = strongly disagree, $2=$ disagree, $3=$ agree, $4=$ strongly agree). Scores are summed across items to obtain a total score. A high score 
Jansen, D.L., Rijken, M., Kaptein, A.A., Boeschoten, E.W., Dekker, F.W., Groenewegen, P.P. The role of social support in dialysis patients' feelings of autonomy and self-esteem: is support more beneficial for patients with specific illness perceptions? Families, Systems \& Health: 2014, 32(3), 313-327

on this scale is indicative of a high level of experienced overprotection. The internal consistency of the scale in this study was 0.85 .

Concern about the illness and perceived personal control over the illness were assessed using the Brief Illness Perception Questionnaire (B-IPQ; Broadbent, Petrie, Main, \& Weinman, 2006). This measure is an abbreviated version of the Revised Illness Perception Questionnaire (IPQ-R Moss-Morris et al., 2002) and consists of nine items each assessing one cognitive or emotional illness representation dimension, including the dimension 'concern' (How concerned are you about your renal disease?) and the dimension 'personal control' (How much control do you feel you have over your renal disease?). The items are scored on an 11-point scale, ranging from 0 to 10 . A higher score on the dimension 'concern' implies greater feelings of concern about the renal disease, and a higher score on the dimension 'personal control' indicates a greater perceived personal control over the renal disease. The B-IPQ has proven to be a reliable and valid measure of illness perceptions in a variety of illness groups (Broadbent et al., 2006).

\section{Background variables.}

Demographic characteristics included age, gender, marital status 2 (married/living together vs. not married/living alone), and educational level (highest level of completed education, classified as low [primary education, lower secondary and lower vocational education], moderate [intermediate secondary and intermediate vocational education], and high [higher vocational education and university]). Clinical characteristics ${ }^{2}$ included time on dialysis (in years), type of dialysis treatment (hemodialysis [HD]/peritoneal dialysis [PD]), and severity of the health condition. Severity of the health condition was determined by the level of serum albumin. Serum albumin is an important predictor of patient morbidity and mortality in dialysis patients (Blake, Flowerdew, Blake, \& Oreopoulos, 1993; Churchill et al., 1992; Plantinga et al., 2007).

\section{Statistical Analyses}

Descriptive statistics were used to describe the background characteristics of the study sample. Differences between the nonresponders and responders regarding sociodemographic and clinical characteristics were tested by means of Student $t$ and chi-square tests.

Descriptive statistics were computed to describe the extent to which dialysis patients experience support and overprotection by their significant others. Interrelationships between the illness perception and support variables were assessed by means of Pearson correlation analyses in order to detect potentially multicollinearity problems. Multiple linear regression analyses were performed, using the enter method, to examine the main and interaction effects of the support and illness perception variables on perceived autonomy and self-esteem. To take possible confounders into account, relevant sociodemographic and clinical characteristics were included in the analyses as control-variables.

We conducted separate regression analyses for each of the three support types combined with either the illness perception 'concern' or 'personal control,' resulting in six regression analyses for each criterion variable (autonomy or self-esteem). Three blocks of variables were entered separately: block 1, sociodemographic variables (age, gender, educational level) and clinical variables (type of dialysis 
Jansen, D.L., Rijken, M., Kaptein, A.A., Boeschoten, E.W., Dekker, F.W., Groenewegen, P.P. The role of social support in dialysis patients' feelings of autonomy and self-esteem: is support more beneficial for patients with specific illness perceptions? Families, Systems \& Health: 2014, 32(3), 313-327

treatment, severity of the health condition); block 2, illness perception variable and support variable; block 3 , cross-product term of the illness perception variable and support variable. Before entering the illness perception and support variables and their cross-product term into the regression analyses, the variables were centered by subtracting the mean score from respondents' raw scores and the product was calculated based upon the centered variables (Aiken \& West, 1991). Centering the continuous variables ensures that the interpretation of effects will occur at a meaningful value of the continuous variable (i.e., the mean, which has a value of 0 with centered variables) and reduces multicollinearity (West, Aiken, \& Krull, 1996). A significant increase in explained variance in the criterion variable as a result of adding the cross-product term to the model, as well as a significant regression coefficient of the product term, indicate an interaction effect. For the present study an interaction effect implies that the relationship between support and autonomy/selfesteem varies with the level of concern/personal control.

\section{RESULTS}

\section{Study Sample}

Characteristics of the total sample are outlined in Table 1. Approximately two thirds of the participants were male, which corresponds with the figures of the population of ESRD patients in the Netherlands (61\%; Renine Foundation, 2005). Around two thirds of the participants were 65 years or older. Compared with figures of the Dutch dialysis population in 2006 (49\% 65 years or older; Oppe, Treur, Barendregt, \& De Charro, 2007), our sample comprises a higher percentage of older patients. A large majority of respondents (71\%) were treated with HD, which is comparable with the percentage HD patients within the Dutch dialysis population in 2005 (74\%; Renine Foundation). No significant differences between the study sample and the nonresponders were found with regard to gender, age, marital status, type of treatment, time on dialysis, and serum albumin level.

\section{[TABLE 1$]$}

\section{Experienced Support}

The mean sum scores of the study sample on the social support interaction scales indicate that patients now and then experience support (see Table 2). Scores found by Van Sonderen (1993) among patients with cancer $(n=399)$ were slightly higher on the general emotional support scale $(M=10.5)$, and slightly lower on the problem-oriented emotional support scale $(M=18.1)$. Scores of the healthy control group $(n=220)$ in the study of Van Sonderen were somewhat lower for both scales (general emotional support: $M=9.8$; problem-oriented emotional support: $M=$ 15.0). In general, dialysis patients experience a low level of overprotection by significant others (see Table 2).

\section{[TABLE 2]}

\section{Relationships Between Support and Illness Perceptions}

The Pearson's correlation coefficients between the support and illness perceptions are small. With respect to concern two significant correlations can be observed. As patients report higher levels of concern about their illness, they report higher levels 
Jansen, D.L., Rijken, M., Kaptein, A.A., Boeschoten, E.W., Dekker, F.W., Groenewegen, P.P. The role of social support in dialysis patients' feelings of autonomy and self-esteem: is support more beneficial for patients with specific illness perceptions? Families, Systems \& Health: 2014, 32(3), 313-327

of problem-oriented emotional support $(r=.22, p<.01)$ and experience more overprotection $(r=.22, p<.01)$. Personal control is not significantly related to support (data not shown).

Hypothesis 1: General and problem-oriented emotional support are positively related to autonomy in patients who report high levels of concern and low levels of personal control (buffering effects).

This hypothesis has been partly confirmed. The results of the regression analyses, in which the emotional support and concern variables are entered, do not demonstrate any significant main effects of general emotional support (see Table 3) or problemoriented emotional support on autonomy (see Table 4).

\section{[TABLE 3 ][TABLE 4 ]}

The results do demonstrate a significant interaction effect $(p=.02)$ of concern and general emotional support on autonomy (Table 3, Model 3). This result indicates that the relationship between general emotional support and autonomy varies with the level of concern. The addition of the product term to the model also leads to a significant contribution in explained variance $\left([\mathrm{DELTA}]_{\mathrm{m} 2,3} R^{2}\right.$ adjusted $=4.3 \%, p=$ .02 ), compared with the model without the product term (Model 2). Next, we examined the nature of the association between general emotional support and autonomy in patients with different concern scores. The $B$ coefficient of general emotional support presented in Table 3 (Model 3) is indicative for people who have mean concern levels (concern score $=0$, because the concern variable is centered). 3 The $B$ coefficient reflects a weak positive $(B=0.01)$, though not significant, association between general emotional support and autonomy. In Figure 1 the relationship is plotted for the highest and lowest concern scores. The results reveal a positive relationship between general emotional support and autonomy in people reporting high levels of concern, and this relationship appears to be significant ( $B=$ $0.09, p=.046)$. Furthermore, the results demonstrate a negative association in people reporting low levels of concern, and this association is close to significant ( $B$ $=-0.13, p=.054)$.

\section{[FIGURE 1]}

The results do not reveal a significant interaction effect of concern and problemoriented emotional support on autonomy (Table 4, Model 3).

Next, we investigated the associations between general emotional support and problem-oriented emotional support, combined with personal control beliefs, on the one hand and autonomy on the other hand. The results do not reveal any significant main or interaction effects (Tables 5 and 6).

\section{[TABLE 5 ] [TABLE 6 ]}

Hypothesis 2a: Problem-oriented emotional support is positively related to selfesteem in patients who report high levels of concern and low levels of personal control (buffering effects).

This hypothesis has been refuted. The results do not reveal significant main effects of problem-oriented emotional support on self-esteem (Tables 7 and 8), and no significant interaction-effects of concern and problem-oriented emotional support on 
Jansen, D.L., Rijken, M., Kaptein, A.A., Boeschoten, E.W., Dekker, F.W., Groenewegen, P.P. The role of social support in dialysis patients' feelings of autonomy and self-esteem: is support more beneficial for patients with specific illness perceptions? Families, Systems \& Health: 2014, 32(3), 313-327

self-esteem (see Table 7) and personal control and problem-oriented emotional support on self-esteem (see Table 8).

[TABLE 7 ][TABLE 8 ]

Hypothesis 2b: General emotional support is positively related to self-esteem in all patients (main effect), and this relationship is stronger in patients who report high levels of concern and low levels of personal control (buffering effects).

This hypothesis has been partly confirmed. The results in Table 9 show that Model 2 , in which the general emotional support and concern variables are added, accounts for a significant increase in explained variance compared to the first model, in which only the background characteristics are entered ([DELTA $]_{\mathrm{m} 1,2} R^{2}$ adjusted $=18.3 \%, p$ $=.000$ ), and also reveal a significant positive main effect of general emotional support on self-esteem (Table 9, Model 2 and 3). The results do not reveal a significant interaction effect of concern and general emotional support on selfesteem.

\section{[TABLE 9]}

Next, we investigated the associations between general emotional support, combined with personal control beliefs, on the one hand and self-esteem on the other hand. The results do not reveal any significant main or interaction effects (see Table 10).

\section{[TABLE 10]}

Hypothesis 3: Overprotection is negatively related to autonomy and self-esteem in all patients (main effects), and these relationships are stronger in patients who report low levels of concern and high levels of personal control (buffering effects).

This hypothesis has been partly confirmed. Table 11 contains the findings regarding the separate and combined relationships of concern and overprotection on autonomy as well as self-esteem. The results with respect to autonomy demonstrate that Model 2 significantly contributes to the explained variance in comparison with the first model ([DELTA $] \mathrm{m}_{1,2} R^{2}$ adjusted $=5.2 \%, p=.03$ ), and reveal a negative significant main effect of overprotection on autonomy (Table 11, Model 2 and 3). The results do not reveal a significant interaction-effect of concern and overprotection on autonomy.

\section{[TABLE 11$]$}

With respect to self-esteem, the results show that the second model accounts for a significant increase in explained variance, compared to the model containing solely the background characteristics ([DELTA $] \mathrm{m}_{1,2} R^{2}$ adjusted $=21.3 \%, p=.000$ ), and that overprotection is significantly negatively associated with self-esteem (Table 11, Model 2 and 3). Again, the results do not reveal a significant interaction-effect of concern and overprotection (on self-esteem).

Finally, we investigated the separate and combined effects of overprotection and personal control on autonomy and self-esteem (see Table 12). With respect to autonomy, the results demonstrate a significant negative main effect of overprotection on autonomy, as well as a significant interaction effect $(p=.03)$ of personal control and overprotection on autonomy (Table 12, Model 3). Model 3, in 
Jansen, D.L., Rijken, M., Kaptein, A.A., Boeschoten, E.W., Dekker, F.W., Groenewegen, P.P. The role of social support in dialysis patients' feelings of autonomy and self-esteem: is support more beneficial for patients with specific illness perceptions? Families, Systems \& Health: 2014, 32(3), 313-327

which the product term is added, accounts for a significant increase in explained variance $\left([\mathrm{DELTA}]_{\mathrm{m} 2,3} R^{2}\right.$ adjusted $\left.=3.2 \%, p=.03\right)$.

\section{[TABLE 12 ]}

The interaction effect indicates that the association between overprotection and autonomy depends on patients' reported personal control levels. Subsequently, we examined the nature of the associations. The B coefficient of overprotection presented in Table 12 (Model 3) shows a negative $(B=-0.02)$ significant relationship $(p<.05)$ between overprotection and autonomy in people with average personal control levels. In Figure 2 the relationship is plotted for the highest and lowest personal control scores. The $B$ coefficients related to the highest and lowest personal control scores demonstrate a significant negative relationship between overprotection and autonomy in people with high levels of personal control $(B=-$ $0.06, p=.003$ ) and a weak positive, though not significant, relationship in people with low levels of personal control $(B=0.01, p=.604)$.

\section{[FIGURE 2]}

With respect to self-esteem, the results show that Model 2 (see Table 12), in which the overprotection and personal control variables are added separately, leads to a significant increase in explained variance, compared with Model 1 ([DELTA $]_{\mathrm{m} 1,2} R^{2}$ adjusted $=12.7 \%, p=.000$ ), and furthermore reveal a negative significant main effect of overprotection on self-esteem (Table 12, Model 2 and 3). The results do not reveal a significant interaction-effect of personal control and overprotection on selfesteem.

\section{Discussion}

In the literature on social support there are two main hypotheses on the role of social support in well-being (Cohen \& Wills, 1985). The first hypothesis proposes that social support is related to well-being only (or primarily) for persons under stress ('stress-buffering'). Cohen, Gottlieb, and Underwood (2000) discuss several pathways through which social support influences individual responses to stressful life events. One of these pathways is that support may alleviate the impact of stress appraisal by providing a solution to the problem, by reducing the perceived importance of the problem, or by providing a distraction from the problem. The second hypothesis argues that social support positively influences well-being irrespective of whether persons face stressful events ('main effect'). This study provides evidence for the existence of stress-buffering effects of support on autonomy, by taking patients' illness representations as stress indicators. Furthermore, we found main effects of support on self-esteem. Below we will discuss the results on the basis of the formulated hypotheses.

\section{Emotional Support and Autonomy}

The findings reveal that the experience of general emotional support is positively related to autonomy, but only in patients with high levels of concern. These results are in accordance with our hypothesis and in line with the stress-buffering hypothesis.

General emotional support was, however, not related to autonomy in people with low levels of personal control. Cohen and Wills (1985) emphasize that support functions 
Jansen, D.L., Rijken, M., Kaptein, A.A., Boeschoten, E.W., Dekker, F.W., Groenewegen, P.P. The role of social support in dialysis patients' feelings of autonomy and self-esteem: is support more beneficial for patients with specific illness perceptions? Families, Systems \& Health: 2014, 32(3), 313-327

have to match with the stressors faced with. Tijhuis et al. (1995) also concluded that social support should be asked specifically, in relation to specific events. General emotional support is relevant to people who experience worries an emotional response-but might not be the most relevant type of support in case people feel that they cannot influence their illness. Perhaps informative support for managing the illness and treatment and integrating the illness and treatment into daily life is more relevant under these circumstances.

In contrast to our expectations, no interaction effect was found of problem-oriented emotional support and illness perceptions on autonomy. The results did not show main effects either. This type of support is only offered in more problematic situations. It could be that this support type cannot boost feelings of autonomy, in light of the problems with which one is faced.

\section{Emotional Support and Self-Esteem}

As expected, we found a positive main effect of general emotional support on selfesteem. As stated before, experienced general emotional support might reflect satisfaction of the general need for belongingness or relatedness and therefore contribute to people's self-esteem. Unlike our expectations, these relationships were however not stronger in patients who reported higher levels of concern and lower levels of personal control. This might reflect a certain limit to the boosting effect of this kind of support, that is, to derive self-esteem through social resources. Contrary to our expectations, no interaction effect was observed of problem-oriented emotional support and illness perceptions on self-esteem. Also here the absence of a positive association between problem-oriented emotional support and self-esteem could be explained by the fact that the problematic situation itself predominates.

\section{Overprotection and Autonomy, Self-Esteem}

We found significant negative associations between overprotection on the one hand and autonomy and self-esteem on the other hand, as hypothesized. In addition, we hypothesized that these relationships would be stronger in people who reported lower levels of concern and higher levels of personal control since these people are less in need of help, let alone unwanted help. This hypothesis was partly confirmed. With respect to autonomy, we found a significant interaction effect indicating that the relationship between overprotection and autonomy varies with the level of personal control. The negative association between overprotection and autonomy appears to be stronger in patients with high levels of perceived personal control over their illness. However, we did not observe a significant interaction effect of overprotection and concern on autonomy. This again may have to do with the linkage of the type of event with the type of support. People high in personal control over the illness in particular believe that they can manage the illness themselves. The experience of overprotection therefore might have an extra negative impact, because it gives the impression that others believe that one is not capable in managing the illness.

It is noteworthy that the present study had a cross-sectional design, which means no conclusions can be drawn with respect to the causality of the observed relationships. For example, a negative relationship between support and autonomy may indicate that experiencing little support results in feeling more autonomous, or that people with a stronger sense of autonomy generate less support from their social environment. Notwithstanding this limitation, our results show that experienced support by patients on dialysis is associated with their feelings of self-esteem, and 
Jansen, D.L., Rijken, M., Kaptein, A.A., Boeschoten, E.W., Dekker, F.W., Groenewegen, P.P. The role of social support in dialysis patients' feelings of autonomy and self-esteem: is support more beneficial for patients with specific illness perceptions? Families, Systems \& Health: 2014, 32(3), 313-327

that illness representations regarding concern and personal control interfere with the relationship between experienced support and feelings of autonomy in patients on dialysis. We were able to reveal significant interaction effects, despite the rather small study sample which reduces the statistical power to demonstrate relationships. With respect to the measurement methods, we like to emphasize that illness perceptions and experienced support were measured via self-reporting, which means that the constructs are assessed from the patients' perspectives. In the first case this is logical, because it concerns patients' personal beliefs about their illness. Received support can however also be measured from the provider's perspective or by observation. However, the interpretation and experience of the patients themselves are the most important and relevant for the outcomes under study.

\section{Implications for Future Practice and Research}

The findings from this study reveal that dialysis patients' experienced social support plays a role in patients' sense of autonomy and self-esteem. Furthermore, the findings show that the role of support in dialysis patients' autonomy depends on patients' perceptions of their illness. These findings suggest that dialysis patients' personal views about their illness can provide insight into whether patients could benefit from support and that the provision of support should be tailored to their individual needs.

We recommend taking these findings into account when developing interventions focused on supporting patients in their efforts to maintain a sense of autonomy and self-esteem. Future research would benefit from using a longitudinal design, which would allow statements about causality and generate recommendations for interventions. We recommend investigating these relationships in other chronic ill patient groups as well.

Furthermore, the results point to the relevance of specifying illness related needs. The results suggest that people who are worried about their illness or who experience little personal control over their illness do not benefit from the same types of support. Regarding these findings, we want to emphasize that our findings only cover the roles of emotional support and overprotection aspects in perceived autonomy and self-esteem. Other types of support exist (e.g., esteem support, instrumental support, informative support) and these support types, combined with the illness perceptions, might deliver different outcomes with respect to autonomy and self-esteem. In addition, the support types and illness perceptions we investigated in the present study might show different relationships with other well-being outcomes, such as vitality or relatedness. More research is needed that focuses on illness perceptions in combination with other support types and/or other aspects of well-being.

\footnotetext{
${ }^{1}$ Patients were recruited on an ongoing basis until January 2007. The cohort was subject to an outflow of patients as a result of mortality or transplantation. [Context Link]

${ }^{2}$ These data were derived from the last known regular NECOSAD-2 measurement at the time of the implementation of the current study. [Context Link]

${ }^{3}$ This is illustrated by the following regression equations: $Y=B 0+0.01 X$ (general emotional support) + - $0.05 Z$ (concern) $+0.02 X Z ; Y=B 0+0.01 X$ (general emotional support) + -0.05 0 (concern) $+0.02 X 0 ; Y=B 0+0.01 X$ (general emotional support). [Context Link]
} 
Jansen, D.L., Rijken, M., Kaptein, A.A., Boeschoten, E.W., Dekker, F.W., Groenewegen, P.P. The role of social support in dialysis patients' feelings of autonomy and self-esteem: is support more beneficial for patients with specific illness perceptions? Families, Systems \& Health: 2014, 32(3), 313-327

\section{REFERENCES}

Aiken, L. S., \& West, S. G. (1991). Multiple regression: Testing and interpreting interactions. Newbury Park, CA: Sage

Baard, P. P., Deci, E. L., \& Ryan, R. M. (2004). Intrinsic need satisfaction: A motivational basis of performance and well-being in two work settings. Journal of Applied Social Psychology, 34, 2045-2068. doi:10.1111/j.1559-1816.2004.tb02690.x

Blake, P. G., Flowerdew, G., Blake, R. M., \& Oreopoulos, D. G. (1993). Serum albumin in patients on continuous ambulatory peritoneal dialysis: Predictors and correlations with outcomes. Journal of the American Society of Nephrology, 3, 1501-1507.

Broadbent, E., Petrie, K. J., Main, J., \& Weinman, J. (2006). The brief illness perception questionnaire. Journal of Psychosomatic Research, 60, 631-637. doi:10.1016/j.jpsychores.2005.10.020

Buunk, B. P., Berkhuysen, M. A., Sanderman, R., Nieuwland, W., \& Ranchor, A. V. (1996). Actieve betrokkenheid, beschermend bufferen en overbescherming. Meetinstrumenten voor de rol van de partner bij hartrevalidatie [Active engagement, protective buffering, and overprotection Measurements for assessing spousal support in heart revalidation]. Gedrag en Gezondheid: Tijdschrift voor Psychologie en Gezondheid, 24, 304-313.

Cameron, L. D., \& Moss-Morris, R. (2004). Illness-related cognition and behaviour. In A. Kaptein \& J. Weinman (Eds.), Health psychology (pp. 84-110). Oxford, UK: Blackwell Publishers.

Churchill, D. N., Taylor, D. W., Cook, R. J., LaPlante, P., Barre, P., Cartier, P., ... Werb, R. (1992). Canadian hemodialysis morbidity study. American Journal of Kidney Diseases, 19, 214-234. doi:10.1016/S0272-6386(13)80002-9

Cohen, S. (2004). Social relationships and health. American Psychologist, 59, 676-684. doi:10.1037/0003-066X.59.8.676

Cohen, S., Gottlieb, B. H., \& Underwood, L. G. (2000). Social relationships and health. In S. Cohen, L. G. Underwood, \& B. H. Gottlieb (Eds.), Social support measurement and intervention: A guide for health and social scientists (pp. 3-25). New York, NY: Oxford University Press.

Cohen, S., \& Wills, T. A. (1985). Stress, social support, and the buffering hypothesis. Psychological Bulletin, 98, 310-357. doi:10.1037/0033-2909.98.2.310

Deci, E. L., La Guardia, J. G., Moller, A. C., Scheiner, M. J., \& Ryan, R. M. (2006). On the benefits of giving as well as receiving autonomy support: Mutuality in close friendships. Personality and Social Psychology Bulletin, 32, 313-327. doi:10.1177/0146167205282148

Deci, E. L., \& Ryan, R. M. (1985). Intrinsic motivation and self-determination in human behavior. New York, NY: Plenum Press. doi:10.1007/978-1-4899-2271-7

Deci, E. L., \& Ryan, R. M. (2000). The "what" and "why" of goal pursuits: Human needs and the self-determination of behavior. Psychological Inquiry, 11, 227-268. doi:10.1207/S15327965PLI1104_01

De Ridder, D., Schreurs, K., \& Kuijer, R. (2005). Is spousal support always helpful in asthma and diabetes? A longitudinal study. Psychology \& Health, 20, 497-508. doi:10.1080/14768320500098699

Ekelund, M. L., \& Andersson, S. I. (2007). Elucidating issues stressful for patients in predialysis and dialysis: From symptom to context. Journal of Health Psychology, 12, 115126. doi:10.1177/1359105307071745

Heatherton, T. F., \& Polivy, J. (1991). Development and validation of a scale for measuring state self-esteem. Journal of Personality and Social Psychology, 60, 895-910. doi:10.1037/0022-3514.60.6.895

Heijmans, M. J. W. M., \& Rijken, P. M. (2004). Sociaal-maatschappelijke participatie van mensen met een chronische nierinsufficiëntie. Een literatuurstudie naar de ervaren knelpunten en mogelijkheden [Social participation of people with chronic renal insufficiency. A literature study on experienced obstacles and opportunities]. Utrecht, The Netherlands: NIVEL.

Hyde, M., Wiggins, R. D., Higgs, P., \& Blane, D. B. (2003). A measure of quality of life in early old age: The theory, development and properties of a needs satisfaction model (CASP-19). Aging \& Mental Health, 7, 186-194. doi:10.1080/1360786031000101157 
Jansen, D.L., Rijken, M., Kaptein, A.A., Boeschoten, E.W., Dekker, F.W., Groenewegen, P.P. The role of social support in dialysis patients' feelings of autonomy and self-esteem: is support more beneficial for patients with specific illness perceptions? Families, Systems \& Health: 2014, 32(3), 313-327

Jansen, D. L., Rijken, M., Heijmans, M., \& Boeschoten, E. W. (2010). Perceived autonomy and self-esteem in Dutch dialysis patients: The importance of illness and treatment perceptions. Psychology \& Health, 25, 733-749. doi:10.1080/08870440902853215 Joekes, K., Van Elderen, T., \& Schreurs, K. (2007). Self-efficacy and overprotection are related to quality of life, psychological well-being and self-management in cardiac patients. Journal of Health Psychology, 12, 4-16. doi:10.1177/1359105306069096

Kasser, V. G., \& Ryan, R. M. (1999). The relation of psychological needs for autonomy and relatedness to vitality, well-being, and mortality in a nursing home. Journal of Applied Social Psychology, 29, 935-954. doi:10.1111/j.1559-1816.1999.tb00133.x

Khan, I. H., Garratt, A. M., Kumar, A., Cody, D. J., Catto, G. R. D., Edward, N., \& MacLeod, A. M. (1995). Patients' perception of health on renal replacement therapy: Evaluation using a new instrument. Nephrology Dialysis Transplantation, 10, 684-689.

Leary, M. R., \& Baumeister, R. F. (2000). The nature and function of self-esteem: Sociometertheory. In M. Zanna (Ed.), Advances in experimental social psychology (Vol. 32, pp. 1-62). San Diego, CA: Academic Press.

Leventhal, H., Meyer, D., \& Nerenz, D. (1980). The common sense representation of illness danger. In S. Rachman (Ed.), Contributions to medical psychology (Vol. 2, pp. 7-30). New York, NY: Pergamon Press.

Leventhal, H., Nerenz, D. R., \& Steele, D. J. (1984). Illness representations and coping with health threats. In A. Baum, S. E. Taylor, \& J. E. Singer (Eds.), Handbook of psychology and health (Vol. 4, pp. 219-252). Hillsdale, NJ: Erlbaum.

Merkus, M. P., Jager, K. J., Dekker, F. W., Boeschoten, E. W., Stevens, P., Krediet, R. T., \& The NECOSAD Study Group. (1997). Quality of life in patients on chronic dialysis: Selfassessment 3 months after the start of treatment. American Journal of Kidney Diseases, 29, 584-592. doi:10.1016/S0272-6386(97)90342-5

Molsted, S., Prescott, L., Heaf, J., \& Eidemak, I. (2007). Assessment and clinical aspects of health-related quality of life in dialysis patients and patients with chronic kidney disease. Nephron Clinical Practice, 106, c24-c33. doi:10.1159/000101481

Moss-Morris, R., Weinman, J., Petrie, K. J., Horne, R., Cameron, L. D., \& Buick, D. (2002). The revised Illness Perception Questionnaire (IPQ-R). Psychology \& Health, 17, 1-16. doi:10.1080/08870440290001494

Oppe, M., Treur, M. J., Barendregt, W., \& De Charro, F. Th. (2007). Statistisch verslag 2007. De ontwikkeling van het nierfunctievervangingsprogramma in Nederland gedurende deperiode 1990-2006 [Statistical report 2007. The development of the renal replacement programme in the Netherlands in the period 1990-2006]. Rotterdam, The Netherlands: Stichting Renine, Renal Replacement Registry Netherlands.

Patel, S. S., Peterson, R. A., \& Kimmel, P. L. (2005). The impact of social support on endstage renal disease. Seminars in Dialysis, 18, 98-102. doi:10.1111/j.1525139X.2005.18203.X

Plantinga, L. C., Fink, N. E., Levin, N. W., Jaar, B. G., Coresh, J., Levey, A. S., ... Powe, N. R. (2007). Early, intermediate, and long-term risk factors for mortality in incident dialysis patients: The Choices for Healthy Outcomes in Caring for ESRD (CHOICE) Study. American Journal of Kidney Diseases, 49, 831-840. doi:10.1053/j.ajkd.2007.03.017

Renine Foundation. (2005). Renal Replacement Registry Netherlands. Rotterdam, The Netherlands. Retrieved August 2007, from http://www.renine.nl/

Ryan, R. M., \& Brown, K. W. (2003). Why we don't need self-esteem: On fundamental needs, contingent love, and mindfulness. Psychological Inquiry, 14, 71-76.

Sarason, B. R., Sarason, I. G., \& Gurung, R. A. R. (1997). Close personal relationships and health outcomes: A key to the role of social support. In S. Duck (Ed.), Handbook of personal relationships: Theory, research, and interventions (2nd ed., pp. 547-573). Chichester, UK: Wiley.

Stel, V. S., van de Luijtgaarden, M. W., Wanner, C., \& Jager, K. J.; on behalf of the European Renal Registry Investigators. (2011). The 2008 ERA-EDTA Registry Annual Report - a précis. NDT Plus, 4, 1-13.

Termorshuizen, F., Korevaar, J. C., Dekker, F. W., Jager, K. J., Van Manen, J. G., Boeschoten, E. W., ... for the NECOSAD Study Group. (2003). Time trends in initiation and dose of dialysis in end-stage renal disease patients in The Netherlands. Nephrology Dialysis Transplantation, 18, 552-558. doi:10.1093/ndt/18.3.552 
Jansen, D.L., Rijken, M., Kaptein, A.A., Boeschoten, E.W., Dekker, F.W., Groenewegen, P.P. The role of social support in dialysis patients' feelings of autonomy and self-esteem: is support more beneficial for patients with specific illness perceptions? Families, Systems \& Health: 2014, 32(3), 313-327

Thompson, S. C., \& Sobolew-Shubin, A. (1993). Perceptions of overprotection in ill adults. Journal of Applied Social Psychology, 23, 85-97. doi:10.1111/j.1559-1816.1993.tb01053.x

Thong, M. S. Y., Kaptein, A. A., Krediet, R. T., Boeschoten, E. W., \& Dekker, F. W., for the NECOSAD Study Group. (2007). Social support predicts survival in dialysis patients. Nephrology Dialysis Transplantation, 22, 845-850. doi:10.1093/ndt/gfl700

Tijhuis, M. A. R., Flap, H. D., Foets, M., \& Groenewegen, P. P. (1995). Social support and stressful events in two dimensions: Life events and illness as an event. Social Science \& Medicine, 40, 1513-1526. doi:10.1016/0277-9536(94)00276-Y

Timmers, L., Thong, M., Dekker, F. W., Boeschoten, E. W., Heijmans, M., Rijken, M., ... for the NECOSAD Study Group. (2008). Illness perceptions in dialysis patients and their association with quality of life. Psychology \& Health, 23, 679-690. doi:10.1080/14768320701246535

Van Sonderen, E. (1993). Het meten van sociale steun met de Sociale Steun Lijst Interacties (SSL-I) en Sociale Steun Lijst Discrepanties (SSL-D), een handleiding [Measuring social support with the Social Support List - Interactions (SSL-I) and Social Support List - Discrepancies (SSL-D-), a manual]. Groningen, The Netherlands: Noordelijk Centrum voor Gezondheidsvraagstukken, Rijksuniversiteit Groningen. [Context Link]

West, S. G., Aiken, L. S., \& Krull, J. L. (1996). Experimental personality designs: Analyzing categorical by continuous variable interactions. Journal of Personality, 64, 1-48.

doi:10.1111/j.1467-6494.1996.tb00813.x

\section{TABLES AND FIGURES}

Table 1

Socio-Demographic and Clinical Characteristics of the Total Sample

\begin{tabular}{|c|c|c|c|}
\hline Characteristic & & Total sample & \\
\hline \multicolumn{4}{|l|}{ Gender $-n(\%)$} \\
\hline Male & 106 & & $(64)$ \\
\hline Female & 59 & & $(36)$ \\
\hline Age, mean in years $(S D)$ & 66.6 & range: $32-89$ & $(11.69)$ \\
\hline \multicolumn{4}{|l|}{ Age, in groups $-n(\%)^{a}$} \\
\hline $18-49$ years & 16 & & $(10)$ \\
\hline $50-64$ years & 46 & & (28) \\
\hline$\geq 65$ years & 104 & & $(63)$ \\
\hline \multicolumn{4}{|l|}{ Educational level - $n(\%)$} \\
\hline Low & 61 & & (39) \\
\hline Moderate & 68 & & (44) \\
\hline High & 27 & & (17) \\
\hline \multicolumn{4}{|l|}{ Marital status $-n(\%)$} \\
\hline Married/living together & 104 & & $(66)$ \\
\hline Not married/living alone & 53 & & (34) \\
\hline \multicolumn{4}{|l|}{ Type of treatment $-n(\%)$} \\
\hline Haemodialysis & 118 & & $(71)$ \\
\hline Peritoneal dialysis & 48 & & $(29)$ \\
\hline Time on dialysis, mean in years $(S D)$ & 3.6 & range: $0.1-9.0$ & $(2.34)$ \\
\hline \multicolumn{4}{|l|}{ Time on dialysis, in groups $-n(\%)$} \\
\hline Up to 2 years & 51 & & $(31)$ \\
\hline 2 to 5 years & 76 & & $(46)$ \\
\hline 5 to 10 years & 39 & & $(23)$ \\
\hline Serum albumin, mean in $\mathrm{g} / \mathrm{L}(S D)^{\mathrm{b}}$ & 36.9 & range: $22.6-45.8$ & $(4.44)$ \\
\hline
\end{tabular}

a Because of rounding the sum of percentages is $>100 \%$. ${ }^{b}$ Normal serum albumin values are $40-50 \mathrm{~g} / \mathrm{L}$. 
Jansen, D.L., Rijken, M., Kaptein, A.A., Boeschoten, E.W., Dekker, F.W., Groenewegen, P.P. The role of social support in dialysis patients' feelings of autonomy and self-esteem: is support more beneficial for patients with specific illness perceptions? Families, Systems \& Health: 2014, 32(3), 313-327

Table 2

Alpha's, Mean Scores, and Standard Deviations of Experienced Emotional Support and Overprotection, Total Sample

\begin{tabular}{lccccrc}
\hline \multicolumn{1}{c}{ Support } & Items & $\alpha$ & Range scale scores & $M(S D)$ & Range scores respondents & $n$ \\
\hline GES & 4 & .82 & $4-16$ & $9.98(2.61)$ & $4-15$ & 158 \\
PES & 8 & .92 & $8-32$ & $18.69(5.12)$ & $8-32$ & 155 \\
Overprotection & 18 & .85 & $18-72$ & $34.50(7.46)$ & $18-60$ & 147 \\
\hline
\end{tabular}

Note. GES = general emotional support; PES = problem-oriented emotional support.

Table 3

Regression Models for Main and Interaction Effects

of Concern and GES on Autonomy $(n=106)$

\begin{tabular}{lcc}
\hline & \multicolumn{2}{c}{ Autonomy } \\
\cline { 2 - 3 } \multicolumn{1}{c}{ Support } & Model 2 & Model 3 \\
\hline Concern & $B(S E)$ & $B(S E)$ \\
GES & $-0.05(0.03)$ & $-0.05(0.03)$ \\
Concern $\times$ GES & $0.01(0.03)$ & $0.01(0.03)$ \\
\hline
\end{tabular}

Note. Controlled for gender, age, educational level (dummy variable: low-moderate, low-high), serum albumin. type of treatment (HD/PD) (Model 1). $B=$ unstandardized coefficient; $S E=$ standard error; GES = general emotional support.

${ }^{*} p<.05$.

\section{Table 4}

Regression Models for Main and Interaction Effects of Concern and PES on Autonomy $(n=106)$

\begin{tabular}{lcc}
\hline \multirow{2}{*}{\multicolumn{1}{c}{ Support }} & \multicolumn{2}{c}{ Autonomy } \\
\cline { 2 - 3 } \multicolumn{1}{c}{ Model 2 } & Model 3 \\
\hline Concern & $B(S E)$ & $B(S E)$ \\
PES & $-0.04(0.03)$ & $-0.04(0.03)$ \\
Concern $\times$ PES & $-0.01(0.02)$ & $-0.01(0.02)$ \\
\hline
\end{tabular}

Note. Controlled for gender, age, educational level (dummy variable: low-moderate, low-high), serum albumin, type of treatment (HD/PD) (Model 1). $B=$ unstandardized coefficient; $S E=$ standard error; $\mathrm{PES}=$ problem-oriented emotional support. 
Jansen, D.L., Rijken, M., Kaptein, A.A., Boeschoten, E.W., Dekker, F.W., Groenewegen, P.P. The role of social support in dialysis patients' feelings of autonomy and self-esteem: is support more beneficial for patients with specific illness perceptions? Families, Systems \& Health: 2014, 32(3), 313-327

Figure 1. Association between general emotional support and autonomy for the highest level and lowest level of concern about the illness (for mean age, male, low educational level, mean serum albumin level, HD treatment).

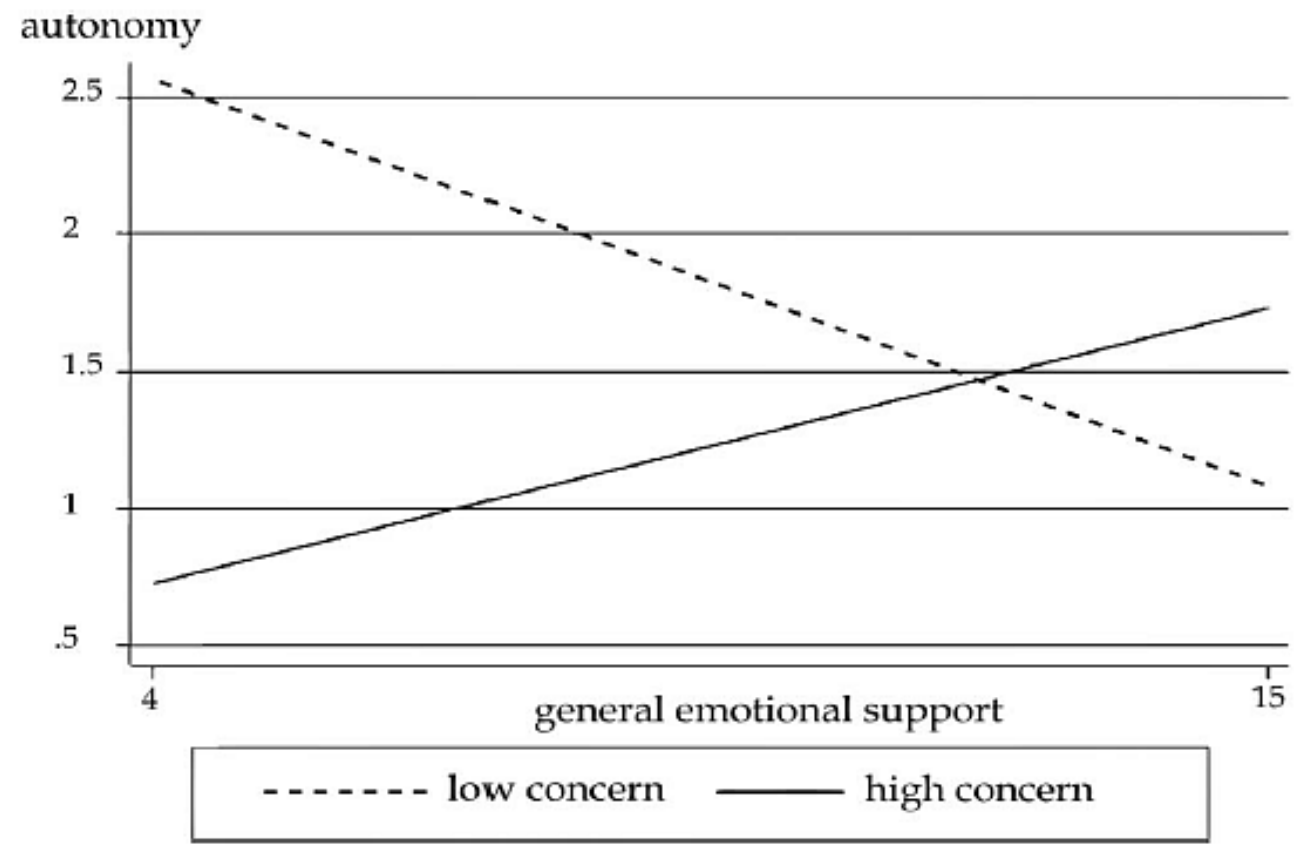

Table 5

Regression Models for Main and Interaction Effects

of Personal Control and GES on Autonomy

$(n=106)$

\begin{tabular}{lcc}
\hline & \multicolumn{2}{c}{ Autonomy } \\
\cline { 2 - 3 } \multicolumn{1}{c}{ Support } & Model 2 & Model 3 \\
& $B(S E)$ & $B(S E)$ \\
\hline Personal control & $0.09(0.02)^{* * *}$ & $0.09(0.02)^{* * *}$ \\
GES & $0.00(0.03)$ & $0.00(0.03)$ \\
Personal control $\times$ GES & & $0.00(0.01)$ \\
\hline
\end{tabular}

Note. Controlled for gender, age, educational level (dummy variable: low-moderate, low-high), serum albumin, type of treatment (HD/PD) (Model 1). $B=$ unstandardized coefficient; $S E$ = standard error; GES = general emotional support.

${ }^{* * * *} p<.001$. 
Jansen, D.L., Rijken, M., Kaptein, A.A., Boeschoten, E.W., Dekker, F.W., Groenewegen, P.P. The role of social support in dialysis patients' feelings of autonomy and self-esteem: is support more beneficial for patients with specific illness perceptions? Families, Systems \& Health: 2014, 32(3), 313-327

Table 6

Regression Models for Main and Interaction Effects of Personal Control and PES on Autonomy $(n=106)$

\begin{tabular}{lcc}
\hline & \multicolumn{2}{c}{ Autonomy } \\
\cline { 2 - 3 } \multicolumn{1}{c}{ Support } & Model 2 & Model 3 \\
& $B(S E)$ & $B(S E)$ \\
\hline Personal control & $0.09(0.02)^{* * *}$ & $0.08(0.02)^{* *}$ \\
PES & $-0.01(0.01)$ & $-0.01(0.01)$ \\
Personal control $\times$ PES & & $0.00(0.01)$ \\
\hline
\end{tabular}

Note. Controlled for gender, age, educational level (dummy variable: low-moderate, low-high), serum albumin, type of treatment (HD/PD) (Model 1). $B=$ unstandardized coefficient; $S E=$ standard error; $\mathrm{PES}=$ problem-oriented emotional support.

${ }^{* *} p<.01 .{ }^{* * *} p<.001$.

Table 7

Regression Models for Main and Interaction Effects of Concern and PES on Self-Esteem $(n=108)$

\begin{tabular}{lcc}
\hline & \multicolumn{2}{c}{ Self-esteem } \\
\cline { 2 - 3 } \multicolumn{1}{c}{ Support } & Model 2 & Model 3 \\
\hline Concern & $B(S E)$ & $B(S E)$ \\
PES & $-1.51(0.34)^{* * *}$ & $-1.51(0.34)^{* * *}$ \\
Concern $\times$ PES & $0.12(0.18)$ & $0.09(0.18)$ \\
\hline
\end{tabular}

Note. Controlled for gender, age, educational level (dummy variable: low-moderate, low-high), serum albumin, type of treatment (HD/PD) (Model 1). $B=$ unstandardized coefficient; $S E$ = standard error; PES = problem-oriented emotional support.

${ }^{* * * *} p<.001$. 
Jansen, D.L., Rijken, M., Kaptein, A.A., Boeschoten, E.W., Dekker, F.W., Groenewegen, P.P. The role of social support in dialysis patients' feelings of autonomy and self-esteem: is support more beneficial for patients with specific illness perceptions? Families, Systems \& Health: 2014, 32(3), 313-327

Table 8

Regression Models for Main and Interaction Effects

of Personal Control and PES on Self-Esteem

$(n=108)$

\begin{tabular}{lcc}
\hline & \multicolumn{2}{c}{ Self-esteem } \\
\cline { 2 - 3 } \multicolumn{1}{c}{ Support } & Model 2 & Model 3 \\
& $B(S E)$ & $B(S E)$ \\
\hline Personal control & $0.80(0.31)^{*}$ & $0.77(0.32)^{*}$ \\
PES & $-0.02(0.19)$ & $-0.02(0.19)$ \\
Personal control $\times$ PES & & $0.03(0.06)$ \\
\hline
\end{tabular}

Note. Controlled for gender, age, educational level (dummy variable: low-moderate, low-high), serum albumin, type of treatment (HD/PD) (Model 1). $B=$ unstandardized coefficient; $S E$ = standard error; $\mathrm{PES}=$ problem-oriented emotional support.

${ }^{*} p<.05$.

Table 9

Regression Models for Main and Interaction Effects of Concern and GES on Self-Esteem $(n=108)$

\begin{tabular}{lcc}
\hline & \multicolumn{2}{c}{ Self-esteem } \\
\cline { 2 - 3 } \multicolumn{1}{c}{ Support } & Model 2 & Model 3 \\
\hline Concern & $B(S E)$ & $B(S E)$ \\
GES & $-1.50(0.31)^{* * *}$ & $-1.50(0.31)^{* * *}$ \\
Concern $\times$ GES & $0.73(0.36)^{*}$ & $0.72(0.36)^{*}$ \\
\hline
\end{tabular}

Note. Controlled for gender, age, educational level (dummy variable: low-moderate, low-high), serum albumin, type of treatment (HD/PD) (Model 1). $B=$ unstandardized coefficient; $S E=$ standard error; GES = general emotional support.

${ }^{*} p<.05 . \quad{ }^{* * *} p<.001$. 
Jansen, D.L., Rijken, M., Kaptein, A.A., Boeschoten, E.W., Dekker, F.W., Groenewegen, P.P. The role of social support in dialysis patients' feelings of autonomy and self-esteem: is support more beneficial for patients with specific illness perceptions? Families, Systems \& Health: 2014, 32(3), 313-327

\section{Table 10}
Regression Models for Main and Interaction Effects
of Personal Control and GES on Self-Esteem
$(n=108)$

\begin{tabular}{lcc}
\hline & \multicolumn{2}{c}{ Self-esteem } \\
\cline { 2 - 3 } & Model 2 & Model 3 \\
\multicolumn{1}{c}{ Support } & $B(S E)$ & $B(S E)$ \\
\hline Personal control & $0.82(0.30)^{* *}$ & $0.82(0.30)^{* *}$ \\
GES & $0.54(0.38)$ & $0.54(0.39)$ \\
Personal control $\times$ GES & & $-0.01(0.12)$ \\
\hline
\end{tabular}

Note. Controlled for gender, age, educational level (dummy variable: low-moderate, low-high), serum albumin, type of treatment (HD/PD) (Model 1). $B=$ unstandardized coefficient; $S E=$ standard error; GES = general emotional support.

${ }^{* *} p<.01$.

Table 11

Regression Models for Main and Interaction Effects of Concern and Overprotection on Autonomy and Self-Esteem $(n=104-107)$

\begin{tabular}{lccccc}
\hline & \multicolumn{2}{c}{ Autonomy } & & \multicolumn{2}{c}{ Self-esteem } \\
\cline { 2 - 3 } \cline { 5 - 6 } \multicolumn{1}{c}{ Support } & Model 2 & Model 3 nnodel 3 & & Model 2 & Mod \\
\hline Concern & $B(S E)$ & $B(S E)$ & & $B(S E)$ & $B(S E)$ \\
Overprotection & $-0.04(0.03)$ & $-0.04(0.03)$ & & $-1.35(0.32)^{* * *}$ & $-1.28(0.33)^{* * *}$ \\
Concern $\times$ Overprotection & $-0.02(0.01)^{*}$ & $-0.02(0.01)^{*}$ & & $-0.36(0.13)^{* *}$ & $-0.37(0.13)^{* *}$ \\
\hline
\end{tabular}

Note. Controlled for gender, age, educational level (dummy variable: low-moderate, low-high), serum albumin, type of treatment (HD/PD) (Model 1). $B=$ unstandardized coefficient; $S E=$ standard error.

${ }^{*} p<.05 .{ }^{* *} p<.01 .{ }^{* * *} p<.001$. 
Jansen, D.L., Rijken, M., Kaptein, A.A., Boeschoten, E.W., Dekker, F.W., Groenewegen, P.P. The role of social support in dialysis patients' feelings of autonomy and self-esteem: is support more beneficial for patients with specific illness perceptions? Families, Systems \& Health: 2014, 32(3), 313-327

Table 12

Regression Models for Main and Interaction Effects of Personal Control and Overprotection on Autonomy and Self-Esteem $(n=104-107)$

\begin{tabular}{|c|c|c|c|c|}
\hline \multirow[b]{2}{*}{ Support } & \multicolumn{2}{|c|}{ Autonomy } & \multicolumn{2}{|c|}{ Self-esteem } \\
\hline & $\begin{array}{c}\text { Model } 2 \\
B(S E)\end{array}$ & $\begin{array}{c}\text { Model } 3 \\
B(S E)\end{array}$ & $\begin{array}{c}\text { Model } 2 \\
B(S E)\end{array}$ & $\begin{array}{c}\text { Model } 3 \\
B(S E)\end{array}$ \\
\hline Personal control & $0.09(0.02)^{* * * *}$ & $0.08(0.02)^{* * *}$ & $0.72(0.30)^{*}$ & $0.71(0.30)^{*}$ \\
\hline Overprotection & $-0.02(0.01)^{*}$ & $-0.02(0.01)^{*}$ & $-0.42(0.13)^{* *}$ & $-0.42(0.13)^{* *}$ \\
\hline Personal control $\times$ Overprotection & - & $-0.01(0.00)^{*}$ & - & $-0.01(0.04)$ \\
\hline
\end{tabular}

Note. Controlled for gender, age, educational level (dummy variable: low-moderate, low-high), serum albumin, type of treatment (HD/PD) (Model 1). $B=$ unstandardized coefficient; $S E=$ standard error.

${ }^{*} p<.05 .{ }^{* *} p<.01 .{ }^{* * *} p<.001$.

Figure 2. Association between overprotection and autonomy for the highest level and lowest level of personal control over the illness (for mean age, male, low educational level, mean serum albumin level, HD treatment).

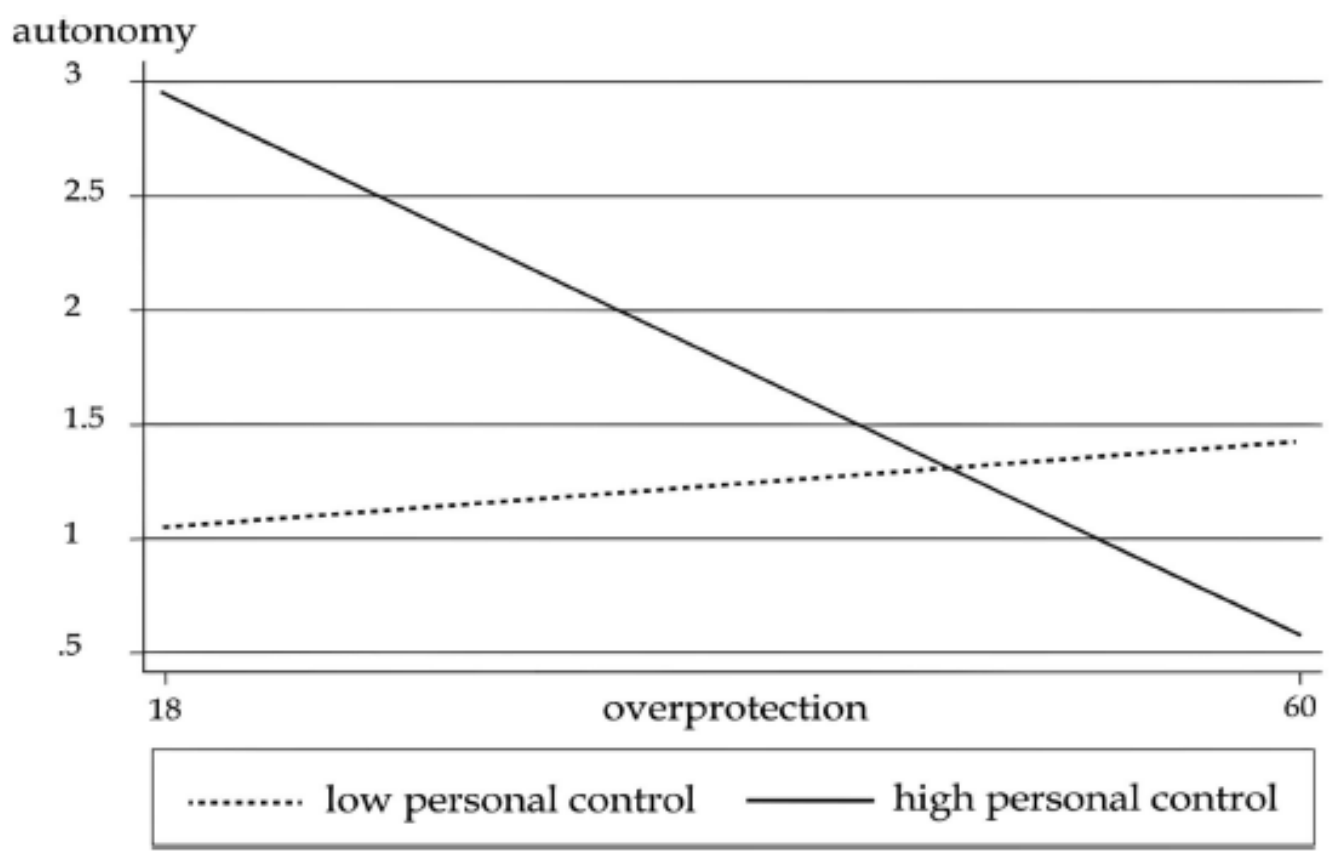

